\title{
Biobanking for Necrotizing Enterocolitis: Needs and Standards
}

\section{Hala Chaaban ${ }^{1}$, Troy A. Markel ${ }^{2}$, Jennifer Canvasser ${ }^{3}$, and Misty Good ${ }^{4 *}$}

${ }^{1}$ Department of Pediatrics, Section of Neonatal-Perinatal Medicine, University of Oklahoma, Oklahoma City, OK, USA

${ }^{2}$ Department of Surgery, Section of Pediatric Surgery, The Indiana University School of Medicine, Indianapolis - Indianapolis, IN

${ }^{3}$ NEC Society, Davis, CA, USA

${ }^{4}$ Department of Pediatrics, Division of Newborn Medicine, Washington University School of Medicine, St. Louis, MO, USA

\section{${ }^{*}$ Correspondence:}

Misty Good, MD, MS

Assistant Professor of Pediatrics

Division of Newborn Medicine

Department of Pediatrics

Washington University School of Medicine

St. Louis Children's Hospital

660 S. Euclid Ave.

Campus Box 8208

St. Louis, MO 63110

Office: 314.286.1329, Fax: 314.286.2897

Email: mistygood@wustl.edu

Funding: HC is supported by grant K08GM127308 from the National Institutes of Health and the University of Oklahoma Health Sciences Center, Department of Pediatrics. TAM is supported by K08DK113226 from the National Institute of Diabetes and Digestive and Kidney Disease of the National Institutes of Health. MG is supported by grants K08DK101608, R03DK111473 and R01DK118568 from the National Institute of Diabetes and Digestive and Kidney Disease of the National Institutes of Health, March of Dimes Foundation Grant No. 5-FY17-79, the Children's Discovery Institute of Washington University and St. Louis Children's Hospital and the Department of Pediatrics at Washington University School of Medicine, St. Louis. None of the funding bodies had any role in the manuscript.

Disclosures: The authors have no conflicts of interest to disclose.

Keywords: Biobank, samples, procurement, barcode, biomarker, biorepository 


\section{Abstract}

Background: Necrotizing enterocolitis (NEC) is a devastating gastrointestinal disease that primarily affects premature infants. Despite medical advances, mortality and morbidity from NEC is still unacceptably high. This is partly due to the lack of specific biomarkers and therapies for this disease. Availability of high-quality biological samples and the associated data from premature infants are key to advance our understanding of NEC, and for biomarker discovery and drug development. To that end, the NEC Society Biorepository was established with the goal of promoting studies in human infants through sharing specialized biospecimen and data procurement for NEC research.

Objective: In this review, we will discuss the required infrastructure for biobanks, discuss the importance of informatics management, and emphasize the logistical requirements for sharing specimens. Finally, we will discuss the mechanism for how tissues and material will be shared between the institutions.

Conclusion: We have developed a state-of-the-art biobank for human infants to advance the field of NEC research. With the NEC Society Biorepository, we seek to facilitate and accelerate the basic and translational studies on NEC to provide hope to the infants afflicted with NEC and their families.

Study type: Review Article, level V

Keywords: Biobank, samples, procurement, barcode, biomarker, biorepository 


\section{Introduction}

\section{Purpose of the necrotizing enterocolitis (NEC) biorepository}

Necrotizing enterocolitis (NEC) is the most common gastrointestinal pathology associated with prematurity, and the mortality remains significant $[1,2]$. Notably, there have been no new advances in diagnostic or therapeutics for NEC in several decades, and as such, the unacceptable clinical outcomes continue to be present. To advance the field of neonatal medicine, and NEC in particular, translational molecular-based studies are required which utilize biological samples from premature neonates. The availability of high quality neonatal biological samples plays a critical role in accelerating the outcomes of translational research studies and provides the foundation for personalized medicine. These neonatal biological samples are housed in biobanks, which are collections of samples collected for research purposes. In 2014, the NEC Society, a non-profit organization, was established with the primary goal of building a world without NEC through research collaborations and improving the broader knowledge base on the scope of the disease. In the first inaugural meeting in 2017, a focus group of researchers and clinicians was convened to consider the merits and challenges of establishing a national biorepository and registry. The group consensus was that a NEC biorepository is essential to advance the state of the science in the hopes to develop novel diagnostic approaches and identify therapeutic targets to prevent and/or treat the disease. This led to the launch of the NEC Society Biorepository [3] in 2018 to serve as an international resource that will provide an opportunity to facilitate collaborations between multiple centers and expand the available number of patients, biological samples and corresponding clinical data. In this 
article, we will first describe the best practices for specimen procurement, we will then highlight our established infrastructure for the NEC biorepository, discuss the importance of informatics management, as well as emphasize the logistical requirements for sharing specimens, data and the ethical implications.

\section{Specimen Procurement and Best Practices}

The NEC Society Biorepository is a newly established biobank of multiple samples including blood, urine, stool, gastric aspirates, resected intestine and genomic DNA from infants with NEC, as well as from appropriate control patients such as those with atresia, ostomy closure, and volvulus. Procurement of the samples requires a trained team of individuals responsible for collecting specimens in an expedited manner with the goal to minimize the ischemic time for the tissue. Capabilities for 24-hour enrollment and sample acquisition are ideal for optimizing specimen integrity and quality.

At our current NEC Biorepository sites, samples are picked up from the NICU at least twice per day and the timing of both collection by nursing as well as delivery to the lab for aliquoting are recorded. For blood and urine collection, samples should be processed and aliquoted immediately and cryopreserved preferably within 4 hours after collection. Data on the type of specimen, date and timing of collection relative to diagnosis of NEC should be included with the samples. For surgically resected intestinal specimens in particular, a procurement service for timely specimen retrieval and aliquoting is essential [4]. This procurement occurs via two different methods depending on the participating center. One approach is to have the specimen taken immediately 
from the operating room to pathology, and the pathologist cuts several aliquots which could be picked up by research investigators for processing, aliquoting, and appropriate storage. The second method in hospital settings where there is not access to 24-hour pathology services to assist with cutting specimens is to utilize a research investigator on-call team, where laboratory members in pediatrics or surgery are called to collect and immediately process the tissues. This processing includes collecting a portion of each sample in an RNA stabilization reagent, a piece fixed in formalin for histologic assessment, and another piece placed in liquid nitrogen snap frozen for analysis at a later date. Importantly, we have learned throughout this process that the intestinal tissue cannot be placed in a refrigerator or freezer and must be processed expeditiously to maintain high-quality samples.

For sample tracking purposes, a barcoding system with consideration of the specific sample location is required for efficient specimen retrieval. A sample storage log with the location, freeze/thaw records, and audit trails of sample acquisition as well as documentation of the restocking of any unused sample is necessary for biorepository quality control.

\section{Infrastructure}

Two main models of biobank networks exist; the federated biobank model where participating centers store their samples and maintain their inventory with capacity for sample sharing; and the centralized biobank model where samples are collected from participating sites and transferred to a central biobank for processing and storage [5]. Infrastructure vary based on the biobank mission, resource scope [6] and adequate 
equipment, staffing, and facilities should be established according to the services to be provided, specimens collected, and researchers served.

In general, requirements for biobanks include ample space with dedicated areas for receiving, processing, storing samples, and shipping if needed. Ideally, space should be located within an academic hospital, or close to neonatal intensive care units (NICUs) and operating rooms, to facilitate access to clinical samples, research support, and minimize the time of sample transfer. Storage equipment selection should be based on the size of the repository, the anticipated number of specimens to be stored, and the funding available. At a minimum, alarmed freezers (-80 degrees Celsius or lower), refrigerators, centrifuge, and computer equipment are required. Newer freezers and refrigerators provide automated sample entry and retrieval which are initially expensive but may reduce long term costs [7]. Liquid nitrogen or -150 degrees Celsius freezers are also recommended for optimal storage of some types of biological material. Where liquid nitrogen is used, daily use should be recorded, and adequate supply maintained. To minimize cost, resource sharing of autoclaves, wet/dry ice, and biological safety cabinets are preferable. Temperature monitoring systems and automated alarm systems should be in place to continuously monitor equipment and warn personnel when equipment failure occurs. In addition, visual inspection of equipment temperatures should be performed regularly, and charts recorded. Backup power sources should be set to activate automatically and should be routinely tested. Adequate backup storage and alternate cooling sources should be available in anticipation of possible equipment failure [8]. For ambient temperature storage, cabinets should be equipped with passive or active humidity controls to maintain biospecimens [9]. 


\section{Biospecimen Sharing}

Translating research from bench to bedside relies significantly on the availability of high quality and clinically annotated biospecimens [10]. Unfortunately, meeting the research needs in NEC will be unachievable by a single biobank due to additional and unique barriers compared to other disease-specific biobanks. These barriers include fewer NEC investigators, difficulty obtaining samples due to emergent operative decisions, and limitations in sample volumes [11, 12]. Therefore, developing NEC biobanking strategies that utilize multiple collection sites combined virtually as seen in the federated model or stored in a centralized biobank, will increase the number and types of specimens available for researchers. In the NEC Society Biorepository, samples are virtually combined by uploading the accompanying information into a shared database. Such models already exist for other disease-specific biorepositories as well and led to the establishment of networks at the international and national level [13-15]. The success of these networks exemplifies the tremendous power of teamwork in science and serves as a model for the future development of projects and enhanced collaboration.

To identify sample availability and access associated clinical data, researchers utilize a NEC Society Biorepository specimen request tool online https://necsociety.org/2018/02/13/nec-society-research-collaborative-launches-necbiorepository-2/, which requires providing the appropriate IRB approval, funding source, and type of specimens or data requested. The researcher is then asked to submit a 1-2page proposal detailing the intent, background information, preliminary data if available, 
and significance of the information requested. The NEC Society Biorepository leadership, composed of a pediatric surgeon, a neonatologist, and a patient-family representative, evaluates the requests to ensure the scientific validity of the research projects, regulatory adherence, and any potential conflicts of interest, then approves or rejects the request. Biospecimen distribution should be based on a defined priority and order of usage for investigators based on the quality of the research project, availability of specimens, and the appropriate controls. Currently, while the NEC Biorepository is in the sample acquisition phase of development, we ask that investigators interested in obtaining samples from our biobank, also join in the efforts of assisting with enrollment and procurement at their center. This will help build up the resources for other investigators and future studies.

\section{Biospecimen shipping and tracking}

The first step before initiation of biospecimen shipment should be determining the regulatory and physical requirements for proper shipping conditions [16, 17]. Shipments should conform to the appropriate governing regulations such as the International Air Transport Association (IATA) standards for air shipments [18]. Most international shipments require additional permits unique to certain countries and need customs clearance displayed on the outside of the package [19]. Shipment of cold or frozen material should be with sufficient and appropriate refrigerant, specifically using dry ice in an insulated container, enough to allow for at least a 24-hour delay in arrival time. For international shipments, three days' worth of refrigerant is recommended in anticipation of possible delays in completing customs requirements. Shipments should be tracked 
by both the shipper and recipient while in transit with an effective tracking system from the collection site through arrival. Components of tracking systems should include the use of unique identifiers or barcodes, specimen labels, and data inventory systems [20, 21]. In addition, the inventory system should track the location of samples in storage, sample movements and status changes, specimen container type, volume or size, time and date of collection, temperature, as well as a preservative. Additional information stored in the inventory varies according to the biospecimens and their intended use but allows for digitally scanned documents to be included such as pathology reports, donor consent forms, and material transfer agreements if applicable.

\section{Informatics and data management}

One of the main obstacles to the progress of biomedical research is the lack of standardized, high-quality data associated with stored samples [5]. Therefore, the strategy for the NEC Society Biorepository has focused on establishing harmonized databases such as a Research Electronic Data Capture (REDCap) for clinical information and integration of the specimen metadata as well as into a database called Open Specimen. The objective being to link adequate data with tissue specimens that are visible to researchers, regardless of their geographical location [8]. The informatics systems at participating centers have the capabilities that allow: 1) inventory functions; 2) data collection and storage; and 3) the ability to query and exchange data between biobanks without compromising security and patient privacy [22]. The inventory system includes tracking of patient enrollment, consent documentation; biospecimen collection, processing, and dissemination; data security; 
and management reporting. The informatics system stores a minimum set of clinical data annotated to the biospecimen. This minimum dataset was determined in consultation with the participating centers and is based on the needs of researchers, as well as overall feasibility and cost. A consensus was reached regarding the terminology used and classification of NEC to harmonize data among all of the participating centers. Interoperability to integrate both clinical and research data as well as distribute biospecimen resources is key to the exchange of data and biospecimens. This interoperability enables integration with local systems and authorized external systems where relevant data can be captured and shared securely. Most importantly, the informatics system storing patient identifiers and protected health information conforms to Health Insurance Portability and Accountability Act (HIPAA) or its equivalent and meets the criteria for National Institute of Standards and Technology (NIST) guidelines [23].

\section{Ethical and legal aspects of biobanks}

Participating biobanks need to adhere to national human subjects' regulations, privacy regulations and other relevant international, national, state and local laws. We acknowledge that a NEC diagnosis is often a traumatic experience for the parents of these fragile neonates. Accordingly, consent must be facilitated in an empathetic, responsive and empowering way. Parents should be provided with as much information as possible in an effort to help families better understand the value of research, what it means to participate, and their ability to help improve outcomes for babies and families 
just like theirs. Parents should be viewed and valued as partners in care and in research. The following additional issues must be considered:

- Informed Consent: Consent is obtained from parents or legal guardians of infants unless waived by an authorized human subjects/ethics committee with applicable laws or regulations [24]. Parents/guardian must be informed of potential genetic analysis on stored material and can opt-out or be excluded from the study. Under 45 CFR 46, if the long-term future use of identified stored biospecimens is likely to constitute greater than minimal risk, biobanks should develop procedures for recontacting participants to obtain the consent of the now-adult participant. Informed consent should contain details on where the samples will be stored, and what information can be shared with other researchers. In certain instances, such as the procurement of deidentified intestinal tissue, the IRB may consider a waiver of consent if appropriate under 45 CFR 46.11(d). Specifically, for the NEC Biorepository, a waiver of consent to obtain deidentified resected intestinal tissue is approved by the IRB at Washington University School of Medicine in St. Louis if families are unavailable for consenting. In addition, investigators should be aware of other competing studies for which patients and families are eligible, and limit the approaches to families during emotionally sensitive times. Investigators could consider rotating which studies for which patients and families are approached, or design a common consent process for similar or related studies to limit the burden on families.

- Privacy: Biobanks should follow well-documented procedures to protect the privacy and confidentiality of infants including assigning a unique code and 
removing all identifying information from the specimens; securely storing specimens and data, and providing firewalls between the subject identity and the recipient investigator.

- Access: Biobanks should develop policies governing information on specimen availability and sharing that is guided by general principles of scientific merit, research qualification, and ethical/legal considerations.

- Material Transfer Agreement (MTA): An agreement with terms consistent with the NIH Data Sharing Policy [25] and other applicable sharing policies, should be used for specimen transfer between organizations. The MTA should include a clear description of the biospecimens, agreement to abide by appropriate laws, rules and regulations; acknowledgments of the recipients' right to further distribute the biospecimens; and description of any expectations regarding the dissemination of research data [26].

\section{Funding}

One of the significant challenges in establishing and maintaining a NEC biobank is securing financial support that can sustain its operations long term. Funding is typically required to cover costs for the emerging research needs, equipment maintenance, personnel salaries, website development, as well as clinical and sample metadata upkeep. Ideally, this will be in the form of funding from local academic/research institutions, extramural grants, philanthropic sources, industry, and/or fees for services also known as recharge. Maintaining and allowing service recharge will help cost recovery and will be fundamental in sustaining the long-term 
operation of the biobank. Notably, the NEC Society Biorepository aims to foster significant collaborations in the field and thus does not currently charge for utilization of the banked specimens.

\section{Future Opportunities and Studies}

Biobanking offers several opportunities for collaborations across institutions nationally and internationally. Specifically, for NEC, these specimens provide opportunities for several potential areas of discovery including biomarker development and genetic susceptibility including newer techniques of genomic, metabolomic, transcriptomic and proteomic approaches, enhancing the field of personalized medicine to neonates. In conclusion, we have developed a state-of-the-art biobank for human infants to advance the field of NEC research. With the NEC Society Biorepository, we seek to facilitate and accelerate the basic and translational studies on NEC to provide hope to the infants afflicted with NEC and their families. 


\section{References}

[1] Patel RM, Kandefer S, Walsh MC, Bell EF, Carlo WA, Laptook AR, et al. Causes and timing of death in extremely premature infants from 2000 through 2011 . The New England journal of medicine 2015;372(4):331-40.

[2] Neu J, Walker WA. Necrotizing enterocolitis. The New England journal of medicine 2011;364(3):255-64.

[3] Ralls MW, Gadepalli SK, Sylvester KG, Good M. Development of the necrotizing enterocolitis society registry and biorepository. Seminars in pediatric surgery 2018;27(1):25-8.

[4] Campbell LD, Betsou F, Garcia DL, Giri JG, Pitt KE, Pugh RS, et al.

Development of the ISBER Best Practices for Repositories: Collection, Storage, Retrieval and Distribution of Biological Materials for Research. Biopreserv Biobank 2012;10(2):232-3.

[5] Somiari SB, Somiari RI. The Future of Biobanking: A Conceptual Look at How Biobanks Can Respond to the Growing Human Biospecimen Needs of Researchers. In: Karimi-Busheri F, editor Biobanking in the 21st Century. Cham: Springer International Publishing; 2015, p. 11-27.

[6] Brisson AR, Matsui D, Rieder MJ, Fraser DD. Translational research in pediatrics: tissue sampling and biobanking. Pediatrics 2012;129(1):153-62.

[7] Vaught J. Developments in biospecimen research. British Medical Bulletin 2015;114(1):29-38.

[8] Vaught J. Biobanking Comes of Age: The Transition to Biospecimen Science. Annual review of pharmacology and toxicology 2016;56:211-28.

[9] Troyer D. Biorepository standards and protocols for collecting, processing, and storing human tissues. Methods Mol Biol 2008;441:193-220.

[10] Hewitt R, Hainaut P. Biobanking in a Fast Moving World: An International Perspective. JNCI Monographs 2011;2011(42):50-1.

[11] Mankoff SP, Brander C, Ferrone S, Marincola FM. Lost in Translation: Obstacles to Translational Medicine. Journal of Translational Medicine 2004;2(1):14.

[12] Buckbee KM. Implementing a pediatric phlebotomy protocol. MLO: medical laboratory observer 1994;26(4):32-5.

[13] Riegman PH, Dinjens WN, Oomen MH, Spatz A, Ratcliffe C, Knox K, et al. TuBaFrost 1: Uniting local frozen tumour banks into a European network: an overview. European journal of cancer (Oxford, England : 1990) 2006;42(16):2678-83.

[14] Yuille M, van Ommen GJ, Brechot C, Cambon-Thomsen A, Dagher G, Landegren $U$, et al. Biobanking for Europe. Briefings in bioinformatics 2008;9(1):14-24.

[15] Tomczak K, Czerwińska P, Wiznerowicz M. The Cancer Genome Atlas (TCGA): an immeasurable source of knowledge. Contemporary Oncology 2015;19(1a):A68-77.

[16] Garcia DL, Bracci PM, Guevarra DM, Sieffert N. International Society for Biological and Environmental Repositories (ISBER) tools for the biobanking community. Biopreserv Biobank 2014;12(6):435-6. 
[17] 2012 best practices for repositories collection, storage, retrieval, and distribution of biological materials for research international society for biological and environmental repositories. Biopreserv Biobank 2012;10(2):79-161.

[18] IATA. International Air Transport Association. Dangerous goods, http://www.iata.org/whatwedo/cargo/dgr/pages/index.aspx; 2017 [accessed 8/24.2017].

[19] IATA. Customs \& Trade Facilitation, http://www.iata.org/whatwedo/cargo/fal/pages/index.aspx; [accessed 8/24.2017].

[20] Voegele C, Alteyrac L, Caboux E, Smans M, Lesueur F, Le Calvez-Kelm F, et al. A sample storage management system for biobanks. Bioinformatics 2010;26(21):2798-800.

[21] Nussbeck SY, Skrowny D, O'Donoghue S, Schulze TG, Helbing K. How to Design Biospecimen Identifiers and Integrate Relevant Functionalities into Your Biospecimen Management System. Biopreserv Biobank 2014;12(3):199-205.

[22] Branch BaBR. NCl Best Practices for Biospecimen Resources., https://biospecimens.cancer.gov/bestpractices/toc/; 2016.

[23] Commerce NloSaTUSDo. Guide for Conducting Risk Assessments http://nvlpubs.nist.gov/nistpubs/Legacy/SP/nistspecialpublication800-30r1.pdf; 2012.

[24] Protections OfHr. Code of Federal Regulations. TITLE 45 PUBLIC WELFARE. PART 46. PROTECTION OF HUMAN SUBJECTS, https://www.hhs.gov/ohrp/regulations-and-policy/regulations/45-cfr-46/index.html; 2009 [accessed 8/31.2017].

[25] Policy NDS. NIH Data Sharing Policy, https://grants.nih.gov/grants/policy/data_sharing/; 2006 [accessed 8/31.2017].

[26] Department HaHS. Simple Letter Agreement for the Transfer of Materials-NIH, https://www.ott.nih.gov/sites/default/files/documents/pdfs/slaform.pdf; 1999 [accessed 8/31.2017]. 